\title{
Opportunistic Vehicular Ferrying for Energy Efficient Wireless Mesh Networks
}

\author{
Keyvan R. Moghadam, Ghada H. Badawy, Terence D. Todd and Dongmei Zhao ${ }^{\dagger}$ \\ Jesús A. Pérez Díaz \\ ${ }^{\dagger}$ Department of Electrical and Computer Engineering \\ McMaster University \\ Hamilton, Ontario, CANADA \\ Email: toddemcmaster.ca \\ $\ddagger$ Department of TI and Mechatronics \\ ITESM - Campus Cuernavaca Xochitepec \\ Morelos, México \\ Email: jesus.arturo.perez@itesm.mx
}

\begin{abstract}
Vehicular ad-hoc networks will soon support a wide variety of inter-vehicle and vehicle-to-roadside applications. In many cases these networks will also co-exist with battery operated networks such as those deployed for sensor and mesh networked applications. In these latter types of networks, mesh node energy efficiency is often of paramount importance. This paper proposes improvements in mesh network energy efficiency by opportunistically ferrying delay tolerant traffic through co-existent vehicular networks. This is accomplished by dynamically routing traffic through vehicular paths when this forwarding is compatible with traffic flow quality-of-service constraints. The proposed mechanism is referred to as opportunistic vehicle assisted forwarding (OVAF). A model is formulated which provides an optimum routing bound under different objectives using hybrid multi-hop mesh routing and vehicular packet forwarding. A heuristic is then presented which can be used in practical networks which use OVAF. Simulation results show large improvements in network performance when this is used compared to conventional singlenetwork multi-hop forwarding.
\end{abstract}

\section{INTRODUCTION}

Vehicular ad-hoc networks (VANETs) will become a major commercial force in the near future. These systems will enable applications ranging from road safety, to those involving context-aware advertising and in-vehicle Internet media streaming. Recognizing the importance of VANETs, the FCC has licensed the operation of dedicated short range communication (DSRC) in the $5.9 \mathrm{GHz}$ frequency band. A new standard for vehicular networks, known as Wireless Access in Vehicular Environment (WAVE), is currently being developed based on the IEEE 802.11 wireless LAN standard.

In many practical situations, VANETs will also co-exist with battery powered networks where energy efficiency is highly desirable. For example, wireless sensor networks are widely used for applications such as environmental monitoring, traffic control, and target tracking. Sensors are typically deployed across a wide area, and collect data that is forwarded to a control center via base stations which are placed inside the deployment area. In another example, outdoor wireless mesh networks are sometimes deployed to provide wireless LAN coverage in locations where wired electricity is not available. In this case, energy sustainable designs based on solar power are often used [1]. In both these examples, the network deployments must be energy efficient and may coexist either partially or completely with operational vehicular networks.

In the above scenario, the energy efficiency of the mesh network may be improved by the opportunistic use of the vehicular network. Vehicles are typically assumed to have unlimited energy reserves since their radio transceivers are operated from a generator which is powered by the vehicle's engine. When vehicles pass through the mesh network deployment region, they may be used to ferry data which would otherwise have to be routed through the battery operated mesh network infrastructure. This forwarding has to be done in a manner that is consistent with the quality of service needed for the forwarded traffic flows.

In this paper we consider the improvements in energy efficiency which are possible by ferrying delay tolerant traffic through co-existent vehicular networks. This is accomplished opportunistically, by dynamically routing traffic through the vehicular network when this forwarding is consistent with the quality of service needed for the forwarded traffic flows. A mechanism referred to as opportunistic vehicle assisted (OVAF) forwarding is introduced and a model is formulated which provides an optimum routing bound under different objective functions using a combination of multi-hop mesh routing and vehicular packet forwarding. Two different objectives are considered. The first minimizes the total mesh network load and the second is based on maximizing the network lifetime. A heuristic is then presented which can be used in practical networks which use OVAF. Simulation results show large improvements in network performance when this is used compared to conventional single-network multi-hop forwarding.

\section{RELATED WORK}

Using mobile objects as message carriers has been extensively used in the context of delay tolerant, and in time, 
wireless sensor networks. In the following discussion we give a brief overview of related work.

The idea of message carrying is used in many delay tolerant network contexts, such as data ferrying, data mules and DakNets[2]. The majority of this work assumes that the message carrying intermittently inter-connects networks which would be otherwise disconnected, rather than using them in a hybrid fashion as considered in this paper.

In many contexts the idea of a mobile base station is used to improve network performance. In [3] multiple base stations are considered which can relocate their positions on the periphery of a sensor network deployment area to help distribute network loading which is concentrated at the base station locations. The authors in [4] devise a path election scheme and adaptive routing for relocating sensor network base stations so that network lifetime can be prolonged. Reference [5] considers a closed trajectory for the base station movement while [6] focuses on event driven base station replacement. The work in this area assumes that the mobility of base stations is again used for fair load distribution and usually considers joint multihop forwarding for improving the network lifetime.

Reference [7] considers different routing schemes for message ferrying in which some mobile nodes carry messages from one sensor island to the others. In [8], message ferries are also used to connect isolated islands in delay tolerant sensor networks which are prone to network partitioning. In [9] a scheme is discussed for choosing the ferry from the sensor nodes and a ferry replacement protocol is proposed when there is partitioning. Data mules with controlled moments are also used in [10] for sparse delay tolerant sensor networks. The authors presented a heuristic for finding the best data mule path such that the required information is collected and returned to the base station. They also consider an optimum scheme in [11], where both data mule message carrying and multihop forwarding is used to minimize message delays subject to energy constraints on the nodes. To the best of our knowledge, vehicular message carriers are used extensively in VANETs such as in [12], but not jointly with delay tolerant sensor networks.

\section{PRoblem Description}

Our problem deals with delay tolerant wireless sensor networks which have access to vehicles moving through their deployment region. Figure 1 shows an example of this type of situation where a vehicular path is given by the dark curve. We assume that the mesh nodes are all battery powered and have a maximum transmit power which imposes a limit on each node's transmission range. As illustrated in the figure, two nodes are assumed to be connected directly when $P_{T, \text { Max }}>$ $P_{T, i j}$, where $P_{T, \text { Max }}$ is the maximum transmission power and $P_{T, i j}$ is the required power for two nodes, $i$ and $j$, to communicate directly. In this paper we assume that $P_{T, i j}$ is given by

$$
P_{T, i j}=b_{1}+b_{2} d_{i j}^{\alpha},
$$

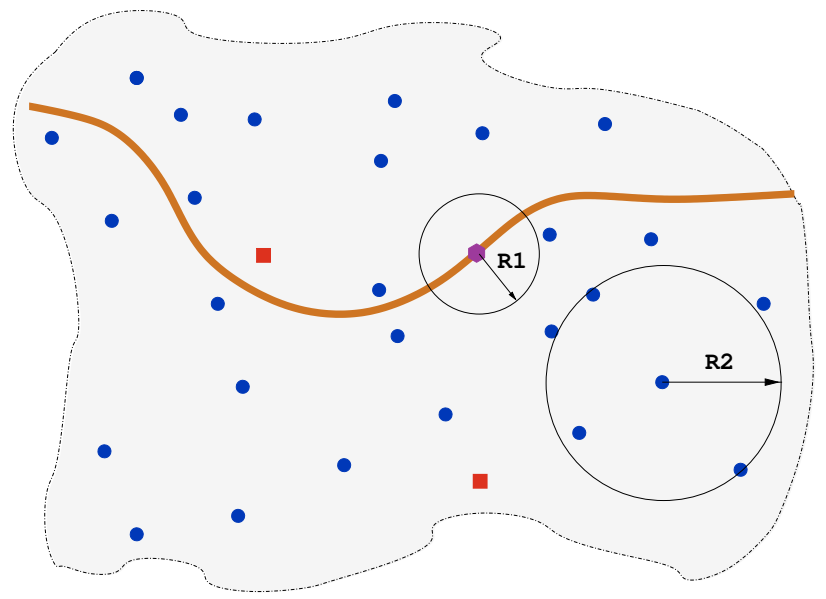

Fig. 1. Wireless Mesh Network with a Co-existent Vehicular Network Path. The vehicular path is shown as a curve. The mesh and base station nodes are shown as circles and squares, respectively.

where $b_{1}$ is a constant which depends on the minimum power required for signal reception and $d_{i j}$ is the distance between Nodes $i$ and $j . b_{2}$ and $\alpha$ model the path loss for the transmitted signal [13] and $2 \leq \alpha \leq 4$ is the exponential path loss coefficient. In our results we assume that $\alpha=2$.

In our network model there are also one or more base stations which collect information and communicate to nodes in the mesh network. These are shown as squares in Figure 1. Network flows are assumed to be unsplittable to reduce undesired behavior such as jitter. It is assumed that the mesh nodes and base stations are aware of the network topology and can adjust their transmit power to use the minimum needed for communicating on each link. We also assume that the mesh nodes can communicate with passing vehicles while the vehicles are within radio coverage range.

We assume that there are delay constraints associated with the packets generated by each flow. The problem is to find the optimum routing as a combination of multi-hop forwarding and vehicular packet forwarding, such that the delivery time of the packets does not exceed their delay constraint, and such that mesh network energy consumption is minimized. We will assume that flows are differentiated based on coarse grained delay tolerance, such that the delay constraints of all the packets in a given flow are satisfied by the chosen route. A route for a particular flow may consist of several different multi-hop and vehicular forwarded segments.

\section{Problem Modeling and Bound Formulation}

In this section we describe the model used in the remainder of the paper and formulate an optimization that can be used to compute a lower bound on the energy efficiency of the mesh network. This bound is compared to a proposed heuristic in Section VI.

The mesh nodes and base stations are identified by integers in the sets $\mathcal{N}:\{1,2, \ldots, N\}$ and $\mathcal{B}:\{1,2, \ldots, B\}$, respectively. Each road position where vehicles can communicate with mesh nodes is defined and indexed by $\mathcal{V}:\{1,2, \ldots, V\}$. 
The mesh network and vehicular carriers are modeled as a graph $G(\mathcal{S}, \mathcal{L})$ in which $\mathcal{S}$ is the set of nodes, each is mapped to each of the members of $\mathcal{N}, \mathcal{B}$, and $\mathcal{V}$ and $\mathcal{L}$ is the set of all pairs $(i, j)$, where $i, j \in \mathcal{S}$, and is defined as

$$
\begin{aligned}
\mathcal{L}=\{(i, j) \mid i, j & \in \mathcal{S} \\
P_{T, i j} & \left.\leq \min \left(P_{T, \operatorname{Max}, i}, P_{T, \text { Max }, j}\right) \text { or } i, j \in \mathcal{V}\right\}
\end{aligned}
$$

where $\mathcal{L}$ indicates the edges of the graph $G . P_{T, M a x, i}$ is the maximum transmission power of Node $i$, for $i \in \mathcal{N} \cup \mathcal{B}$, and when $i \in \mathcal{V}$, it is the maximum vehicular transmit power. By this definition all the nodes which are able to communicate directly have an edge in the graph. It is obvious that all the nodes in $\mathcal{V}$ are able to communicate with each other directly through vehicular message carrying.

For each link in the graph, a delay stamp, $D_{i j}(t)$, is defined which gives the expected maximum delay for delivering data directly from Node $i$ to Node $j$ in each time slot $t$. When $i \in$ $\mathcal{N} \cup \mathcal{B}$ and $j \in \mathcal{V}$, this includes the waiting time for finding a vehicle for forwarding, when $i, j \in \mathcal{V}$ this is the maximum time for a vehicle to travel from Position $i$ to Position $j$, and in the other cases it is the comparatively short time to directly relay the packet from one node to another.

In the formulation we also define a binary variable, $a_{i j}$, as

$$
a_{i j}= \begin{cases}1 & (i, j) \in \mathcal{L} \\ 0 & \text { otherwise }\end{cases}
$$

Now, we incorporate the flow continuity, available bandwidth of each link and power consumption of each node into the formulation. For each possible mesh network flow, we assign an integer in the set $\mathcal{F}:\{1,2, \ldots, F\}$ while $\operatorname{Source}(k)$ and Dest $(k)$ are functions giving the source and destination of Flow $k$, respectively, and $F l w(k, t)$ is the corresponding flow volume at time slot $t$. The flow continuity for each Node $i \in \mathcal{N} \cup \mathcal{V}$ can be written as

$$
\begin{aligned}
\sum_{j \in \mathcal{S}} x_{i j}^{k}(t) a_{i j}- & \sum_{j \in \mathcal{S}} x_{j i}^{k}(t) a_{j i}= \\
& \begin{cases}F l w(k, t) & i=\operatorname{Source}(k) \\
-F l w(k, t) & i=\operatorname{Dest}(k) \\
0 & \text { otherwise. }\end{cases}
\end{aligned}
$$

where $x_{i j}^{k}(t)$ is the portion of Flow $k$ which is routed from Node $i$ to $j$ in time slot $t$. The first term of the left hand side of Equation (1) is the total outgoing volume of Flow $k$ from Node $i$ at time slot $t$, and the second term on the left hand side is the total incoming volume of Flow $k$ at time slot $t$. The right hand side is the volume of Flow $k$, which is generated in Node $i$ and is $-F l w(k)$ if it is the flow destination and zero otherwise. Since all of the base stations are modeled as a single node, the flows destined for, or originated from, the base stations can be sent out from or delivered to each. The flow continuity constraint for the base stations can be written as

$$
\begin{gathered}
\sum_{i \in \mathcal{B}}\left(\sum_{j \in \mathcal{N} \cup \mathcal{V}} x_{i j}^{k}(t) a_{i j}-\sum_{j \in \mathcal{N} \cup \mathcal{V}} x_{j i}^{k}(t) a_{j i}\right)= \\
\left\{\begin{array}{lll}
F l w(k, t) & \text { if } & \operatorname{Source}(k) \in \mathcal{B} \\
-F l w(k, t) & \text { if } & \operatorname{Dest}(k) \in \mathcal{B} \\
0 & \text { otherwise. }
\end{array}\right.
\end{gathered}
$$

To account for link capacity constraints, the total amount of traffic which is routed through each link should not exceed the capacity of the channel, i.e.,

$$
0 \leq \sum_{k \in \mathcal{F}} x_{i j}^{k}(t) \leq C_{i j}, \quad(i, j) \in \mathcal{L}
$$

where $C_{i j}$ is the normalized available bandwidth for Link $(i, j)$. The summation gives us the total amount of the traffic which is routed through Node $i$ to $j$. As stated, the flows are assumed to be inseparable. Thus we define a decision variable $u_{i j}^{k}$ to force this assumption, i.e., $u_{i j}^{k}$ is a binary variable defined as

$$
u_{i j}^{k}(t)=\left\{\begin{array}{lll}
1 & \text { if } & x_{i j}^{k}(t)>0,(i, j) \in \mathcal{L} \\
0 & \text { if } & x_{i j}^{k}(t)=0
\end{array}\right.
$$

The inseparability of flows for each Flow $k$ and Node $i$ is modeled by

$$
\begin{gathered}
\sum_{j \in \mathcal{S}} u_{i j}^{k}(t) a_{i j} \leq 1 \\
\sum_{i \in \mathcal{B}}\left(\sum_{j \in \mathcal{N} \cup \mathcal{V}} u_{i j}^{k}(t) a_{i j}\right) \leq 1
\end{gathered}
$$

The first inequality indicates that flows cannot split while traveling from each Node $i \in \mathcal{N}, \mathcal{V}$. The first term in Inequality (6) is the portion of a specific Flow $k$ coming from the set of basestations and guarantees flow inseparability. The load on each Node $i \in \mathcal{N}$ at time $t$ can be computed by using the modeling we introduced so far, i.e.,

$$
\begin{gathered}
L_{i}(t)=\sum_{k \in \mathcal{F}} \sum_{j \in \mathcal{S}}\left(x_{i j}^{k}(t) a_{i j} P_{t, i j}+x_{j i}^{k}(t) a_{j i} P_{r, i j}\right) \\
+P_{i d l e} \sum_{j \in \mathcal{S}}\left(a_{i j} I_{i j}(t)+a_{j i} I_{j i}(t)\right) \\
I_{i j}(t)=\frac{C_{i j}-\sum_{k \in \mathcal{F}} x_{i j}^{k}(t)}{C_{i j}}
\end{gathered}
$$

The first term on the right hand side of Equation (7) is the total energy which Node $i$ uses at time $t$ to transmit and receive, and the second term is the amount of energy during idle periods, for time $t$. The idle period is obtained by computing the utilization factor of a link in Equation (8).

We assume that the total maximum delay of a flow from source to its destination is the sum of the maximum delay stamps of the links that the flow is routed through. This total expected delay must be less than the expected delivery time of the flow which is primarily determined when it is sent out from the source based on its priority. This constraint for each Flow $k \in \mathcal{F}$ can be mathematically stated as follows

$$
\sum_{(i, j) \in \mathcal{L}} u_{i j}^{k}(t) D_{i j}(t) \leq y^{k}(t) \quad \forall k \in \mathcal{F}
$$


in which $y^{k}(t)$ is the delay constraint for Flow $k$ in time slot $t$. The left hand side of Inequality (9) calculates the summation of all the delay stamps of the links which are participating in the routing of Flow $k$ at time slot $t$. The mesh nodes are assumed to be battery powered and their battery usage is modeled in discrete time. The network lifetime is divided into time slots and the amount of remaining energy for each node, $B_{i}(t)$, is updated at the end of each time epoch, according to the energy recursion

$$
B_{i}(t)=\max \left(B_{i}(t-1)-L_{i}(t), B_{\text {outage }}\right), \quad i \in \mathcal{N} .
$$

It can be seen that Equation (4) is not linear, but can be linearized as follows.

$$
u_{i j}^{k}(t) \in\{0,1\}, \quad x_{i j}^{k}(t) \leq u_{i j}^{k}(t)<1+x_{i j}^{k}(t)
$$

The above model gives a linear mixed-integer optimization. Two different objectives are used. The first minimizes the total energy usage of the network and is formulated as follows.

$$
\operatorname{minimize} \sum_{i \in \mathcal{N}} \sum_{t} L_{i}(t)
$$

subject to:

$$
\begin{aligned}
& u_{i j}(t) \in\{0,1\} \\
& 0 \leq \sum_{k \in \mathcal{F}} x_{i j}^{k}(t) \leq C_{i j}, \quad(i, j) \in \mathcal{L} \\
& x_{i j}^{k}(t) \leq u_{i j}^{k}(t)<1+x_{i j}^{k}(t) \\
& \sum_{j \in \mathcal{S}} u_{i j}^{k}(t) a_{i j} \leq 1 \\
& \sum_{i \in \mathcal{B}}\left(\sum_{j \in \mathcal{N} \cup \mathcal{V}} u_{i j}^{k}(t) a_{i j}\right) \leq 1 \\
& \sum_{j \in \mathcal{S}} x_{i j}^{k}(t) a_{i j}-\sum_{j \in \mathcal{S}} x_{j i}^{k}(t) a_{j i}= \\
& \left\{\begin{array}{lll}
F l w(k, t) & \text { if } & i=\operatorname{Source}(k) \\
-F l w(k, t) & \text { if } & i=\operatorname{Dest}(k) \\
0 & & \text { otherwise. }
\end{array}\right. \\
& \sum_{i \in \mathcal{B}}\left(\sum_{j \in \mathcal{N} \cup \mathcal{V}} x_{i j}^{k}(t) a_{i j}-\sum_{j \in \mathcal{N} \cup \mathcal{V}} x_{j i}^{k}(t) a_{j i}\right)= \\
& \left\{\begin{array}{lll}
F l w(k, t) & \text { if } & \operatorname{Source}(k) \in \mathcal{B} \\
-F l w(k, t) & \text { if } & \operatorname{Dest}(k) \in \mathcal{B} \\
0 & & \text { otherwise. }
\end{array}\right. \\
& \sum_{(i, j) \in \mathcal{L}} u_{i j}^{k}(t) D_{i j}(t) \leq y^{k}(t) \quad \forall k \in \mathcal{F} \\
& L_{i}(t)=\sum_{k \in \mathcal{F}} \sum_{j \in \mathcal{S}}\left(x_{i j}^{k}(t) a_{i j} P_{t, i j}+x_{j i}^{k}(t) a_{j i} P_{r, i j}\right) \\
& +P_{\text {idle }} \sum_{j \in \mathcal{S}}\left(a_{i j} I_{i j}(t)+a_{j i} I_{j i}(t)\right)
\end{aligned}
$$

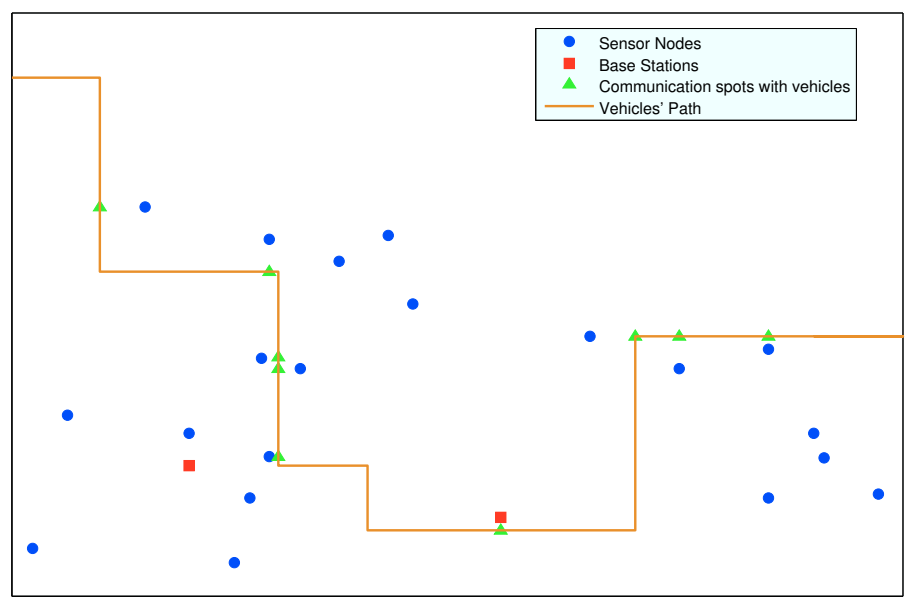

Fig. 2. 20 Node Network Example with 2 Basestations and a Predefined Vehicular Path

$$
\begin{gathered}
I_{i j}(t)=\frac{C_{i j}-\sum_{k \in \mathcal{F}} x_{i j}^{k}(t)}{C_{i j}} \\
B_{i}(t)=B_{i}(t-1)-L_{i}(t), \quad i \in \mathcal{N} \\
B_{\text {outage }} \leq B_{i}(t)
\end{gathered}
$$

The routing with this first objective is equivalent to shortest path routing if we define the length of the path as the distance that flows traverse in the mesh network, i.e. omitting the distance that they are carried by the vehicles. If we consider network lifetime as the time when the first node depletes its battery reserves, the second objective can be formulated as

$$
\operatorname{minimize} \max _{i \in \mathcal{N}} \sum_{t} L_{i}(t)
$$

to maximize lifetime, and which replaces the objective function in Equation (11). This minimizes the maximum battery usage of the nodes and extends the lifetime of the network.

The above formulation results in an optimum bound which can be used as a basis for comparison with practical forwarding algorithms. An algorithm for this is proposed in the next section.

\section{Heuristic Forwarding Algorithm}

It can be seen that the optimization in Section IV is a linear integer program involving a large number of integer decision variables, making it infeasible for large problem instances. In addition, the optimization is in general a nonrealizable bound since the node traffic flows are considered in a non-causal manner. To address this issue, we also propose a heuristic which can be used in practical networks. In this greedy algorithm, the optimal route is designed based on the current and previous data flows for the current time slot. An optimization is used in each time slot, and therefore the size of the problem is equal to the number of time slots, $T$. For the first objective, i.e., shortest path, the algorithm solves the same optimization as in Section IV with the deployment lifetime of 1 time slot and using the traffic flow and flow delay constraints 


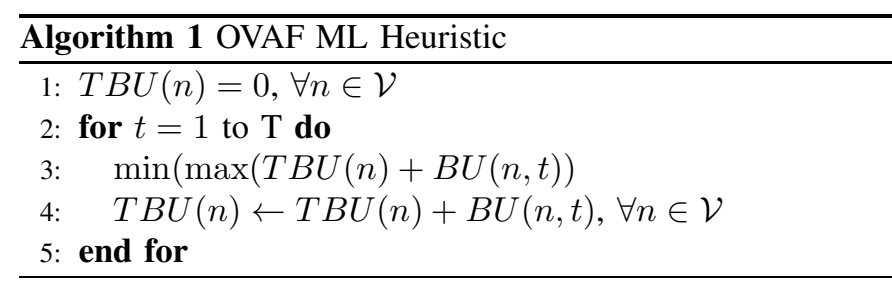

during that time slot. For the second objective we consider the algorithm shown as Algorithm 1, where $T B U(n)$ is the total battery usage (i.e., energy consumed) for Node $n$ in the previous time slots and $B U(n, t)$ is an array of variables indicating the battery (i.e., energy) usage of each node in the time epoch, $t$. This algorithm contains a loop which solves an optimization for each time slot. In each iteration, a set of the variables corresponding to the battery usage of the nodes in the current time slot is determined by solving the optimization problem. The objective, which is minimized is the maximum battery usage of the nodes at the end of the current time slot given the battery usage of the nodes in previous time slots.

\section{Simulation Model and Results}

In this section we present simulation results which evaluate the performance of the proposed algorithms. The simulation is performed for mesh networks with different numbers of nodes that originate from, and are destined to the base stations. Each flow in each time slot has a delay constraint which is randomly chosen from a uniform distribution between 0 and $y_{\max }$ and has a normalized value of 0.5 . A path of vehicles is also defined with a predefined traffic pattern. The expected waiting time for each node in the communication range of vehicles and the expected carrying times of the vehicles are computed based on this traffic pattern.

Figure 2 shows an example of a network with 20 mesh nodes and 2 basestations. The nodes are randomly placed using a uniform distribution within the network area. The solid line indicates the vehicular path and the positions marked by triangles on the path indicate the corresponding nodes in $\mathcal{V}$. The simulations are performed for mesh networks with 10 , 15, 20, 30 and 40 nodes and the two different objectives, i.e., Shortest path (SP), Maximum lifetime (ML), and the heuristic for both of these objectives as previously discussed.

The simulation results are compared with the results of conventional mesh networks which do not use opportunistic vehicular forwarding. We refer to this as Multihop Mesh Forwarding (MMF) in the remainder of the paper. We use the same two objectives, i.e., Shortest path (SP) and Maximum lifetime (ML) for MMF routing.

The accumulated load of each node under four different scenarios for a 10 node mesh network is shown in Figures 3 and 4, which give the results for the first hundred hours of network deployment time. The optimization results using SP are shown in Figure 3 and the results for ML in Figure 4. Most of the nodes have to forward much less load in vehicle assisted forwarding and it can be seen that the loads are more evenly distributed. Node seven has a very low load burden in both

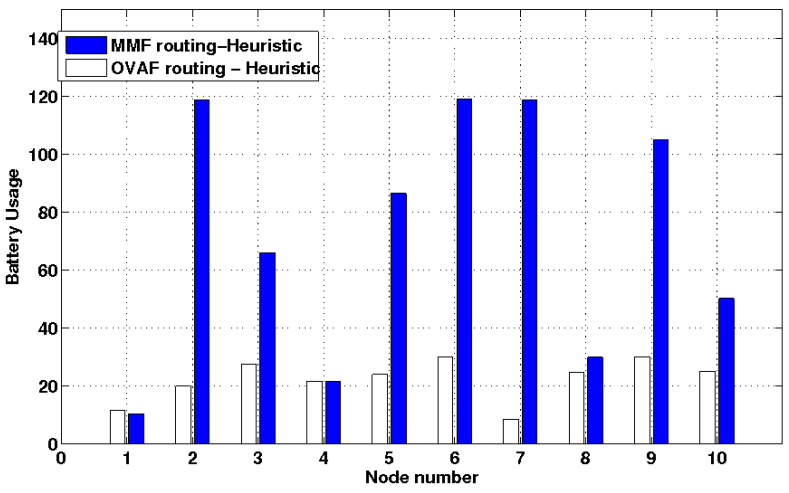

Fig. 3. Battery Usage in a 10 Mesh Node Network after 100 Hours of Deployment Time Using the SP Objective.

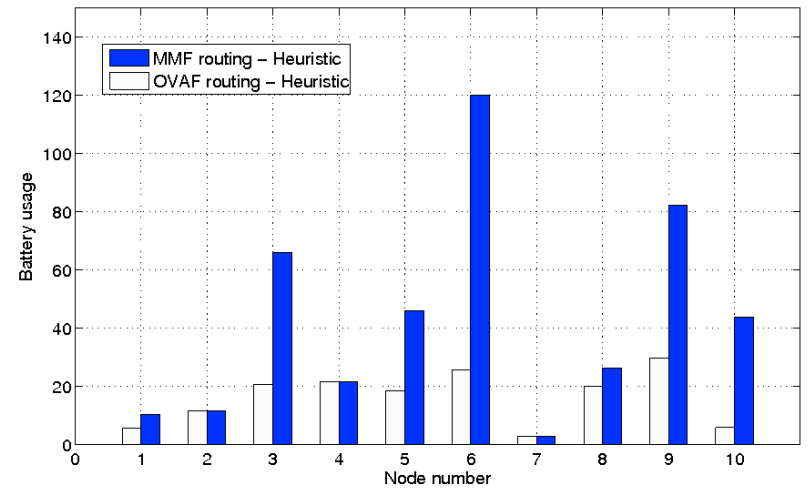

Fig. 4. Battery Usage in a 10 Mesh Node Network after 100 hours of Deployment Time Using the ML Objective.

since it does not participate in multi-hop mesh forwarding. With the ML objective, the mesh network has to support a higher average load at the expense of a longer network lifetime. With the ML objective, higher numbers of nodes deplete at the end of the network lifetime compared to the SP objective. It can also be seen that loading is more evenly distributed with the ML objective than the SP objective for both MMF and OVAF routing.

The average load is compared in Figure 5 for both the lower bounds and the heuristic with two objectives and higher numbers of nodes after 100 hours of deployment. For the SP objective, vehicular forwarding saves more than $60 \%$ compared to the energy used in MMF. ML uses much more resources for both the vehicular and non-vehicular cases, since its objective is to minimize the maximum use of resources. The fair load distribution in vehicular forwarding is also notable in the ML objective.

Figure 6 shows the maximum battery usage of the heuristic and lower bound for ML objective and can be seen how close the heuristic results are to the lower bound. An interesting observation by comparing the two figures is how much more average energy the LB has to use in MMF routing to slightly 


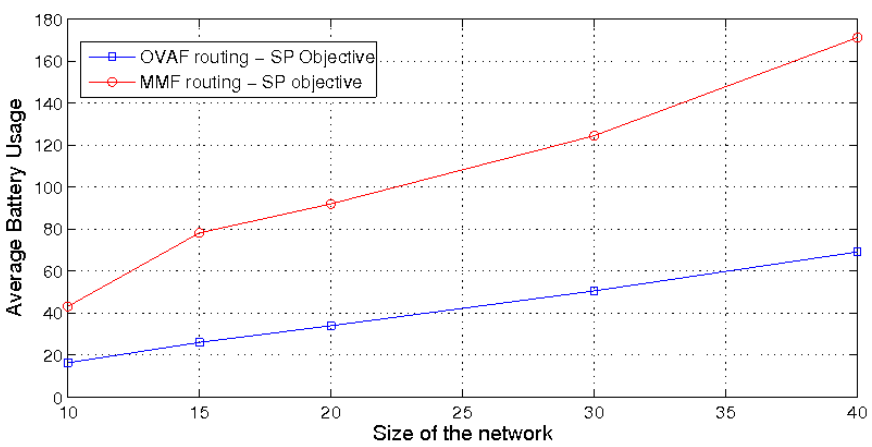

(a) SP Objective

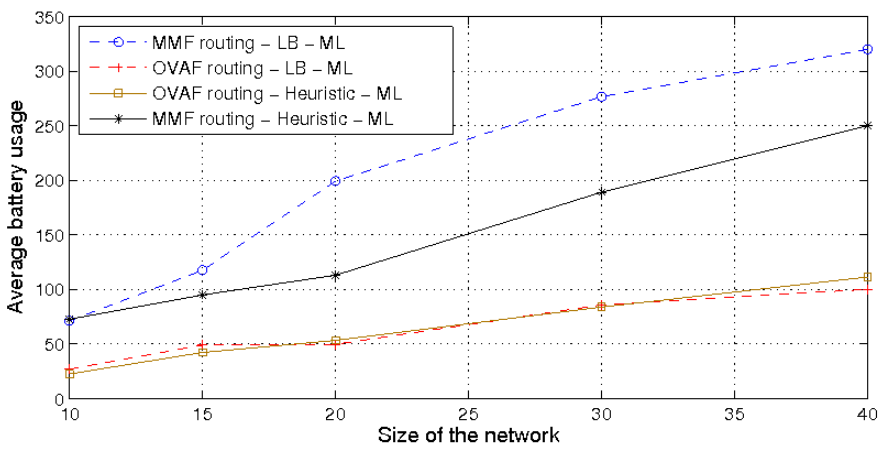

(b) ML Objective

Fig. 5. Average Battery Usage

improve the ML objective while in OVAF routing the average energy usage of both LB and the heuristic are almost the same. It is also notable that the heuristic generates the same result as the lower bound for the SP objective since this objective does not need to use the other time slot flow information to find the shortest path in each time epoch.

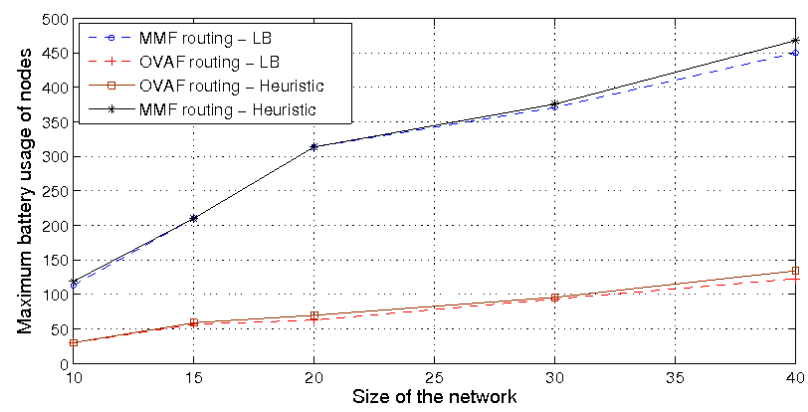

Fig. 6. Maximum battery usage for different network sizes after 100 hours of deployment for ML objective

\section{CONCLUSION}

In this paper we have proposed improvements in mesh network energy efficiency by opportunistically ferrying delay tolerant traffic through co-existent vehicular networks. This is done by routing traffic through vehicular paths when this forwarding is consistent with traffic flow quality-of-service constraints. A model was formulated which obtains an optimum routing bound under both shortest path (SP) and maximum lifetime (ML) objectives. Simulation results of a proposed heuristic showed large improvements in network performance when this is used compared to conventional single-network multi-hop forwarding. In particular, we find that using the ML criterion leads to much improved network lifetimes compared to that using the SP criterion and that obtained without vehicular forwarding. It was also found that the proposed algorithm results in considerable improvements in fair load distribution.

\section{REFERENCES}

[1] A. A. Sayegh, T. D. Todd, and M. N. Smadi, "Resource Allocation and Cost in Hybrid Solar/Wind Powered WLAN Mesh Nodes," Wireless Mesh Networks: Architectures and Protocols, Springer Science and Business Media Inc., pp. 167-189, 2008.

[2] A. Pentland, R. Fletcher, and A. Hasson, "DakNet: Rethinking Connectivity in Developing Nations," Computer, vol. 37, no. 1, pp. 78-83, 2004.

[3] S. Gandham, M. Dawande, R. Prakash, and S. Venkatesan, "Energy Efficient Schemes for Wireless Sensor Networks with Multiple Mobile Base Stations," in Proceedings of IEEE Globecom, vol. 1, 2003, pp. 377-381.

[4] K. Akkaya and M. Younis, "Energy-aware delay-constrained routing in wireless sensor networks," International Journal of Communication Systems, vol. 17, no. 6, pp. 663-687, 2004.

[5] J. Luo and J. Hubaux, "Joint Mobility and Routing for Lifetime Elongation in Wireless Sensor Networks," in Proceedings of IEEE INFOCOM 2005, vol. 3, 2005, pp. 1735-1746.

[6] M. Zhao, Z. Chen, X. Deng, L. Zhang, A. Liu, and G. Huang, "MAEC: A Movement-Assisted Energy Conserving Method in Event Driven Wireless Sensor Networks," in 2005 International Conference on Wireless Communications, Networking and Mobile Computing, vol. 2, 2005, pp. 915-920.

[7] Y. Zhu, W. Wu, and V. Leung, "Energy-Efficient Tree-Based Message Ferrying Routing Schemes for Wireless Sensor Networks," Mobile Networks and Applications, pp. 1-13, 2008.

[8] W. Zhao, M. Ammar, and E. Zegura, "Controlling the Mobility of Multiple Data Transport Ferries in a Delay-Tolerant Network," in Proceedings of IEEE INFOCOM 2005, vol. 2, 2005, pp. 1407-1418.

[9] J. Yang, Y. Chen, M. Ammar, and C. Lee, "Ferry Replacement Protocols in Sparse Manet Message Ferrying Systems," in 2005 IEEE Wireless Communications and Networking Conference, vol. 4, 2005, pp. 20382044.

[10] R. Sugihara and R. Gupta, "Improving the Data Delivery Latency in Sensor Networks with Controlled Mobility," Distributed Computing in Sensor Systems, pp. 386-399, 2008.

[11] _ - "Optimizing Energy-Latency Trade-Off in Sensor Networks with Controlled Mobility," in Proceedings of IEEE INFOCOM 2009, 2009, pp. 2566-2570.

[12] A. Skordylis and N. Trigoni, "Delay-Bounded Routing in Vehicular AdHoc Networks," in Proceedings of the 9th ACM International Symposium on Mobile Ad Hoc Networking and Computing. ACM, 2008, pp. 341350 .

[13] Y. Shi and Y. Hou, "Approximation Algorithm for Base Station Placement in Wireless Sensor Networks," in 4th Annual IEEE Communications Society Conference on Sensor, Mesh and Ad Hoc Communications and Networks, 2007. SECON'07., 2007, pp. 512-519. 